\title{
In vivo reflectance confocal microscopy features of a melanoacanthoma
}

\author{
Neda Shahriari ${ }^{1}$, Jane M. Grant-Kels ${ }^{1}$, Harold S. Rabinovitz ${ }^{2}$, Margaret Oliviero ${ }^{2}$, Alon Scope ${ }^{3}$
}

1 Department of Dermatology, University of Connecticut Health Center, Farmington, CT, USA

2 Department of Dermatology, University of Miami Miller School of Medicine, Miami FL, USA

3 Department of Dermatology, Sheba Medical Center and Sackler Faculty of Medicine, Tel Aviv University, Tel Aviv, Israel

Key words: melanoacanthoma, RCM, microscopy, seborrheic keratosis

Citation: Shahriari N, Grant-Kels JM, Rabinovitz HS, Oliviero M, Scope A. In vivo reflectance confocal microscopy features of a melanoacanthoma. Dermatol Pract Concept 2016;6(4):6. doi: 10.5826/dpc.0604a06

Received: July 21, 2016; Accepted: August 27, 2016; Published: October 31, 2016

Copyright: $@ 2016$ Shahriari et al. This is an open-access article distributed under the terms of the Creative Commons Attribution License, which permits unrestricted use, distribution, and reproduction in any medium, provided the original author and source are credited.

Funding: None.

Competing interests: The authors have no conflicts of interest to disclose.

All authors have contributed significantly to this publication.

Corresponding author: Jane M. Grant-Kels, MD, UCONN Dermatology Dept, 21 South Rd, Farmington, CT 06032, USA. Email: grant@ uchc.edu

ABSTRACT Efforts have been expended to evaluate the reflectance confocal microscopy (RCM) features of different clinical entities in order to more thoroughly delineate benign versus malignant features. In this way, RCM can help clinicians to be more selective in regard to undertaking appropriate skin biopsies and improving their benign to malignant ratio. Herein, we report a case of a histopathologically proven melanoacanthoma, a variant of seborrheic keratosis. There are scarce reports describing the RCM features of melanoacanthoma. Our case demonstrated RCM features that were suspicious for melanoma. More RCM images of this benign entity are needed to establish definitive diagnostic criteria.

\section{Case presentation}

A 51-year-old woman presented with a $7 \mathrm{~mm}$ black papule on the left submandibular region (Figure 1A). The papule was irregularly shaped, sharply demarcated and with a verrucous surface (Figure 1B). The patient had noted the lesion only five weeks earlier and reported it had been growing rapidly. Dermoscopic evaluation revealed blue-white and black clods and scale (Figure 2). The differential diagnosis at this point included nodular melanoma that would have required an excisional biopsy; however, a pigmented seborrheic keratosis, which could be sampled with a shave biopsy, was considered due to the stuck-on, verrucous appearance of the lesion [1]. Therefore, reflectance confocal microscopy (RCM) of the lesion was undertaken. The overall architecture was that of ridges and surface depressions filled with keratotic debris, echoing the clinically observed verrucous surface (Figure 3). Within the hyperplastic epidermis, including the suprabasal layers, there were numerous tangled dendritic cells (Figure 4), suggestive of melanocytes in a pagetoid distribution throughout the epidermis. Based on RCM examination, a melanoma was suspected and the lesion was therefore excised. Histopathological analysis revealed an exophytic, well-circumscribed silhouette, the reticulated, hyperplastic epidermis showed compact hyperkeratosis, acanthosis and horn pseudocysts, intermingled with the keratinocytes throughout the lesion were numerous large, dendritic, melanin-rich melanocytes (Figure 5 A, B, C) confirmed with MART-1 staining 

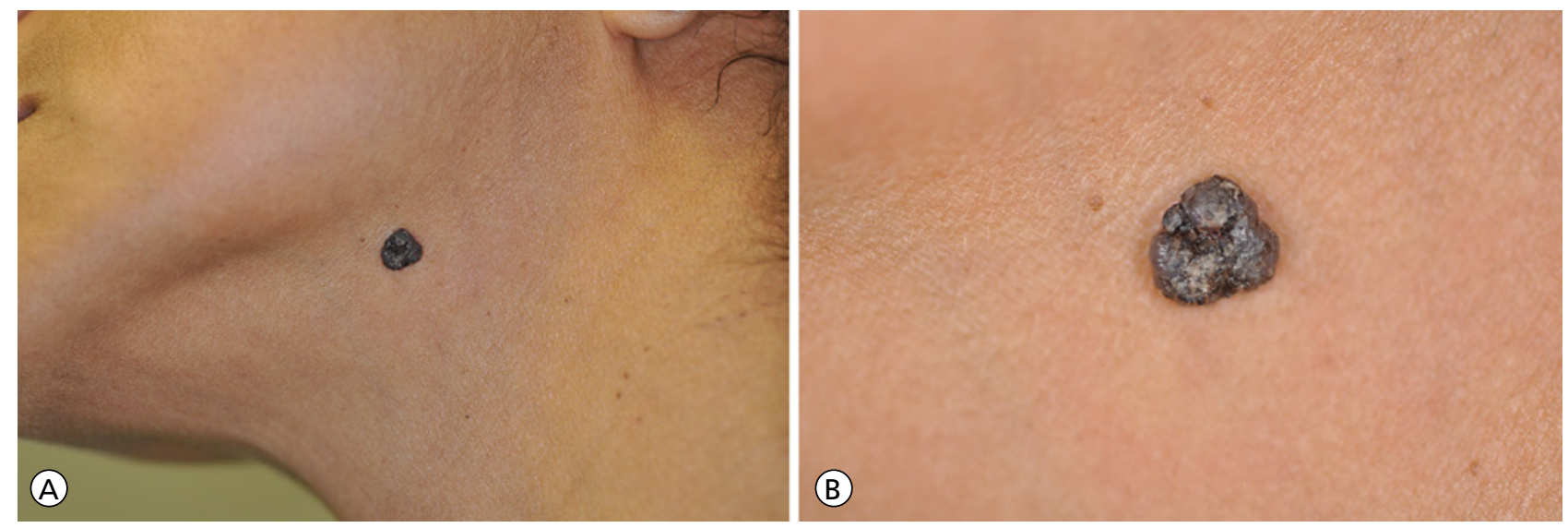

Figure 1. Clinical photographs. (A) Pigmented papule on the left submandibular region (B) The irregular, 7 mm black papule displays a verrucous surface. [Copyright: @2016 Shahriari et al.]

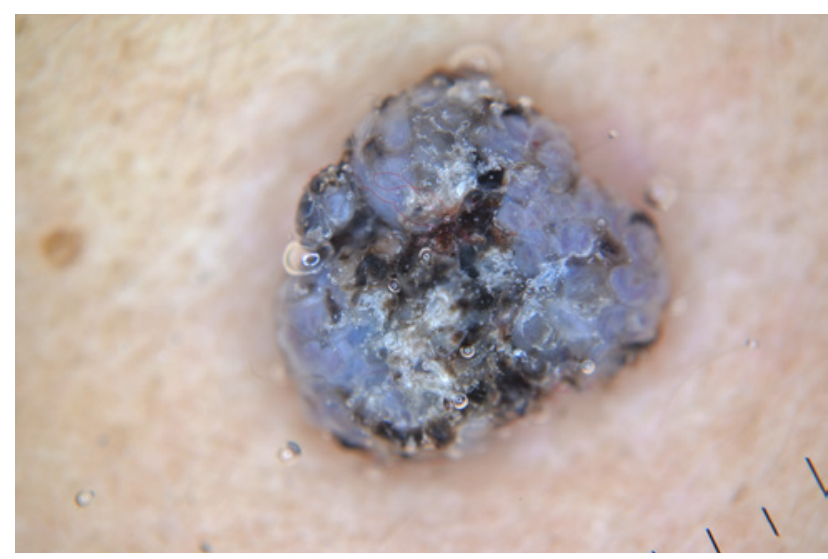

Figure 2. Dermoscopic image shows a verrucous contour with irregular blue-white and black clods. [Copyright: (02016 Shahriari et al.]

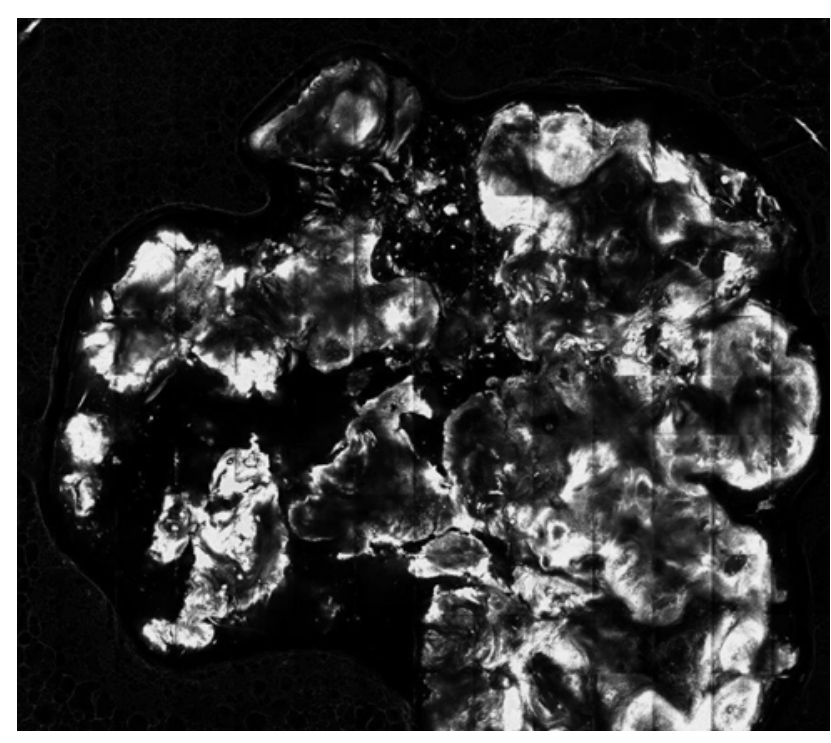

Figure 3. RCM image of the lesion at the stratum corneum layer demonstrates a well-circumscribed lesion with overall architecture of ridges and surface depressions filled with keratotic debris. [Copyright: (2016 Shahriari et al.]

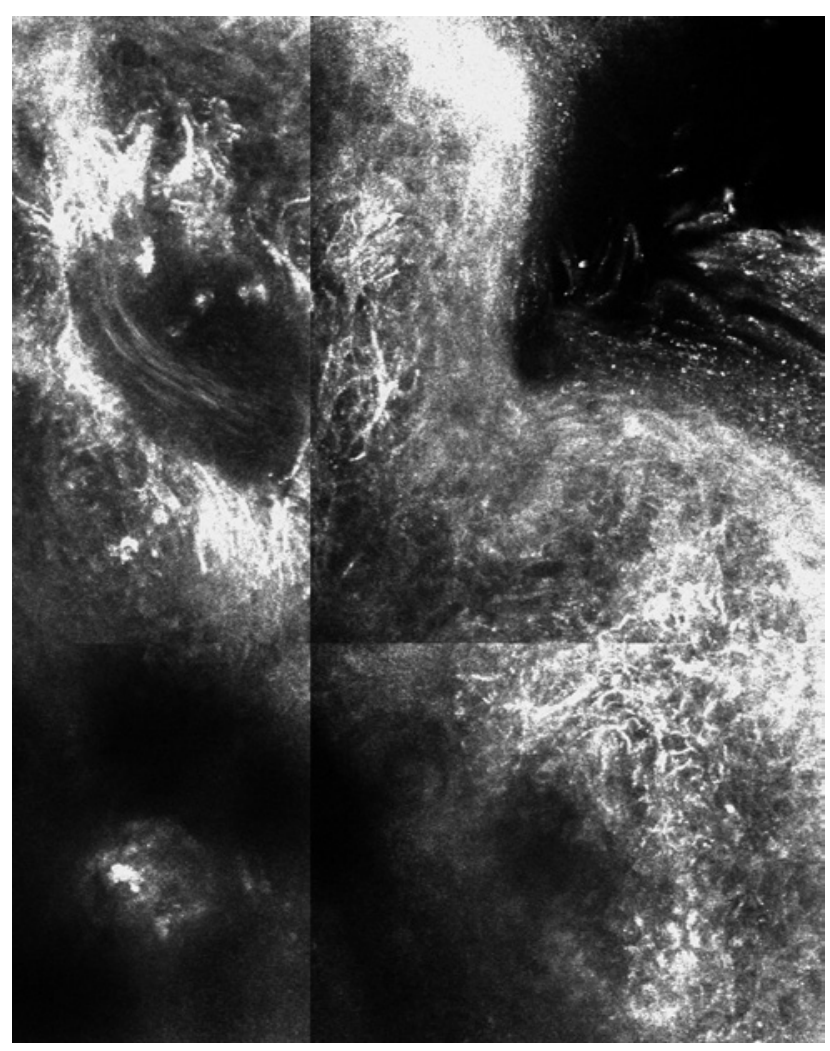

Figure 4. RCM image of lesion $\left(500 \times 500 \mathrm{um}^{2}\right)$ at the spinous granular layers of the epidermis show numerous tangled bright dendritic cells. [Copyright: @2016 Shahriari et al.]

and consistent with the diagnosis of seborrheic keratosis, melanoacanthoma variant.

\section{Discussion}

Seborrheic keratosis (SK) is a benign epidermal proliferation commonly found in individuals in the fifth decade of life and older [2]. Classically, SK is readily identified as a papule or plaque with a greasy "stuck-on" appearance, sharply demarcated borders and brown-tan color. SKs may appear on the head, trunk and extremities, but always spare the 

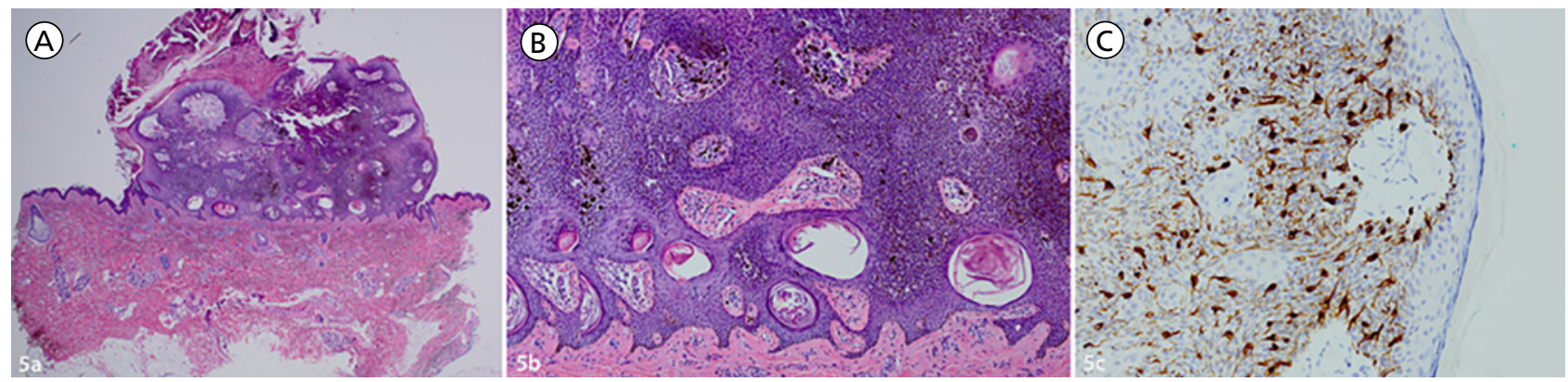

Figure 5. Histopathology of the lesion shows an exophytic, well-circumscribed, reticulated epidermal proliferation confined to a markedly widened papillary dermis. The hyperplastic epidermis shows compact hyperkeratosis, acanthosis and horn pseudocysts, findings diagnostic for seborrheic keratosis. Intermingled with the keratinocytes throughout the epidermis are numerous large, dendritic, melanin-rich melanocytes confirmed with MART-1 staining. [Copyright: @2016 Shahriari et al.]

palmo-plantar surfaces. In addition to this classic appearance, SK can present many morphological "faces"; in fact, some SKs may be quite difficult to clinically distinguish from other diagnostic entities, such as squamous cell carcinoma, melanocytic nevi, or even malignant melanomas [3,4]. On histopathology, SK can be divided into six principle variants: acanthotic, hyperkeratotic, adenoid, irritated, clonal and melanoacanthoma [5].

Melanoacanthoma, a term coined by Mishima and Pinkus [6], is an infrequent variant of SK that is clinically notable for its dark pigmentation, making this entity difficult to distinguish from melanoma at the bedside [7]. Histopathologically, melanoacanthoma shows an epidermis usually marked by hyperkeratosis, acanthosis and horn pseudocysts, as well as islands of small basaloid cells intermingled with large dendritic melanocytes. The melanocytes are large, dendritic, contain abundant melanin, and are not restricted to the basal cell layer [8,9].

Melanoacanthoma has been scarcely characterized with dermoscopy or with RCM. One case of histopathologically confirmed melanoacanthoma demonstrated under dermoscopy the starburst pattern, which is usually seen in pigmented Spitz/Reed nevi [10]. Another report of melanoacanthoma identified a cribriform pattern of ridges [11]. A more recent case series reported on the dermoscopic features of eight histopathologically proven melanoacanthomas. All eight lesions had at least one dermoscopic feature associated with SK, including comedo-like openings, sharp demarcation, more than two milia-like cysts, ridges and fissures, moth-eaten borders, or hairpin vessels [12]. However, six of these lesions also showed dermoscopic features worrisome for melanoma, which would have likely prompted a biopsy.

Dermoscopic evaluation of the lesion reported herein did not show any clear-cut features of SK or a starburst pattern. In fact, the clinically and dermoscopically observed blueblack pigmentation prompted us to consider the diagnosis of a pigmented nodular melanoma [13,14]. Nodular melanomas may lack the classic ABCD criteria for melanoma diagnosis
$[13,14]$; therefore, the "EFG" rule, which assesses "elevation," "firmness" on palpation, and continuous "growth" over one month, was established to more critically assess lesions of this subtype [15]. More recently, the use of the "blue-black rule" was prompted as an important clue for the diagnosis of pigmented nodular melanoma [16,17]. As such, the benign lesion described herein would raise suspicions for melanoma.

To this end, we utilized RCM imaging to identify distinguishing features between melanoacanthoma and pigmented nodular melanoma in hopes of sparing unnecessary excisional biopsy of a benign lesion. We could not identify previous publications reporting the RCM features of melanoacanthoma. In the present case, we observed by RCM the ridges and surface depression that reflect the verrucous surface but could not observe other RCM criteria for SK, such as widening and interweaving of the rete ridges ("polycylic papillary contours") and horn pseudocysts [18]. It is likely that the thickened keratotic surface epidermis hindered imaging of the DEJ. We did observe numerous, tangled dendritic cells, at the basal and supra-basal layers of the epidermis, which we interpreted as suspicious for a melanoma. Of note, we did not identify any other RCM criteria of nodular melanoma-including a thinned epidermis with flattening of the dermo-epidermal junction (DEJ), disarranged epidermis, pleomorphic cells with bright cytoplasm at the basal layer and in pagetoid distribution, and "cerebriform" nests in the papillary dermis [19,20]. In hindsight, the dense, almost uniform proliferation of dendritic melanocytes at both the basal and suprabasal layers of the epidermis may be a clue to melanoacanthoma; however, this potential clue may be difficult to evaluate in cases where the basal layer is not explorable. On the other hand, previous reports of the presence of dendritic cells in RCM of melanomas were typically observed in flat lesions [21,22]. Therefore, another possible diagnostic clue for the RCM of melanoacanthoma may be the presence of dendritic cells in the context of a thickened epidermis with RCM characteristics consistent with seborrheic keratosis, including fissures and horn pseudocysts. 
In summary, in the melanoacanthoma variant of SK presented herein, ruling out melanoma proved difficult on clinical and dermoscopic examination, as well as by RCM imaging. More cases of melanoacanthomas imaged by RCM will be needed to try and establish specific RCM criteria for the diagnosis of this benign entity.

\section{References}

1. Longo C, Farnetani F, Ciardo S, et al. Is confocal microscopy a valuable tool in diagnosing nodular lesions? A study of 140 cases. Br J Dermatol 2013;169:58-67. PMID: 23374159. DOI: 10.1111/bjd.12259.

2. Gill D, Dorevitch A, Marks R. The prevalence of seborrheic keratoses in people aged 15 to 30 years: is the term senile keratosis redundant? Arch Dermatol 2000;136(6):759-62. PMID: 10871940.

3. Marks R, Jolley D, McCormack C, Dorevitch AP. Who removes pigmented skin lesions? J Am Acad Dermatol 1997;36:721-6. PMID: 9146533. DOI: 10.1016/S0190-9622(97)80324-6.

4. Longo C, Moscarella E, Piana S, et al. Not all lesions with a verrucous surface are seborrheic keratoses. J Am Acad Dermatol 2014;70:e121-3. PMID: 24831328. DOI: 10.1016/j. jaad.2013.10.042.

5. Roh NK, Hahn HJ, Lee YW, Choe YB, Ahn KJ. Clinical and histopathological investigation of seborrheic keratosis. Ann Dermatol 2016;28(2):152-8. PMID: 27081260. DOI: 10.5021/ ad.2016.28.2.152.

6. Mishima Y, Pinkus H. Benign mixed tumor of melanocytes and malpighian cells. Melanoacanthoma: Its relationship to Bloch's benign non-nevoid melanoepithelioma. Arch Dermatol 1960;81:539-50. PMID: 14422903.

7. Bolognia JL, Jorizzo JL, Rapini RP, eds. Dermatology. Philadelphia, PA: Mosby Elsevier, 2008:1661-4.

8. Schlappner OL, Rowden G, Phillips TM, Rahim Z. Melanoacanthoma. Ultrastructural and immunological studies. J Cutan Pathol 1978;5:127-41. PMID: 210197. DOI: 10.1111/j.16000560.1978.tb00949.x.

9. Kirkham N. Tumors and cysts of the epidermis. In: Elder DE (ed.). Lever's Histopathology of the Skin. 10th ed. New Delhi: Lippincott, 2009:791-849.

10. Rossiello L, Zalaudek I, Ferrara G, et al. Melanoacanthoma simulating pigmented Spitz nevus: an unusual dermoscopy pitfall. Dermatol Surg 2006;32(5):735-7. PMID: 16706772. DOI: 10.1111/j.1524-4725.2006.32148.x.
11. Shankar V, Nandi J, Ghosh K, Ghosh S. Giant melanoacanthoma mimicking malignant melanoma. Indian J Dermatol 2011;56(1):79-81. PMID: 26083708. DOI: 10.1001/jamadermatol.2015.1453.

12. Chung E, Marghoob AA, Carrera C, Marchetti MA. Clinical and dermoscopic features of cutaneous melanoacanthoma. JAMA Dermatol 2015;151(10):1129-30. PMID: 26083708. DOI: 10.1001/jamadermatol.2015.1453.

13. Liu W, Dowling JP, Murray WK, et al. Rate of growth in melanomas: characteristics and associations of rapidly growing melanomas. Arch Dermatol 2006;142:1551-8. PMID: 17178980. DOI: 10.1001/archderm.142.12.1551.

14. Clark WH Jr, From L, Bernardino EA, Mihm MC. The histogenesis and biologic behaviour of primary human malignant melanomas of the skin. Cancer Res 1969;29:705-27. PMID: 5773814.

15. Kalkhoran S, Milne O, Zalaudek I, et al. Historical, clinical, and dermoscopic characteristics of thin nodular melanoma. Arch Dermatol 2010;146:311-18. PMID: 20231503. DOI: 10.1001/ archdermatol.2009.369.

16. Argenziano G, Longo C, Cameron A, et al. Blue-black rule: a simple dermoscopic clue to recognize pigmented nodular melanoma. Br J Dermatol 2011;165(6):1251-5. PMID: 21916885. DOI: $10.1111 / \mathrm{j} .1365-2133.2011 .10621 . x$.

17. Pizzichetta MA, Kittler H, Stanganelli I, et al. Pigmented nodular melanoma: the predictive value of dermoscopic features using multivariate analysis. Br J Dermatol 2015;173(1):106-14. PMID: 25916655. DOI: 10.1111/bjd.13861.

18. Ahlgrimm-Siess V, Cao T, Oliviero M, et al. Seborrheic keratosis: reflectance confocal microscopy features and correlation with dermoscopy. J Am Acad Dermatol 2013;69:120-6. PMID: 23415460. DOI: 10.1016/j.jaad.2012.12.969.

19. Segura S, Pellacani G, Puig S, et al. In vivo microscopic features of nodular melanomas: dermoscopy, confocal microscopy, and histopathologic correlates. Arch Dermatol 2008; 144:1322-20. PMID: 18936395. DOI: 10.1001/archderm.144.10.1311.

20. Pupelli G, Ferrari B, Farnetani F, et al. Melanoma. In: Gonzalez S (ed.). Reflectance Confocal Microscopy in Dermatology: Fundamentals and Clinical Applications. Aulamedica, 2012:47-51.

21. Pellacani G, De Pace B, Reggiani C, et al. Distinct melanoma types based on reflectance confocal microscopy. Exp Dermatol 2014;23:414-8. PMID: 24750486. DOI: 10.1111/exd.12417.

22. Braga JC, Macedo MP, Pinto C, et al. Learning reflectance confocal microscopy of melanocytic skin lesions through histopathologic transversal sections. PLoS One 2013;8:e81205. PMID: 24339910. DOI: 10.1371/journal.pone.0081205. 